\title{
Dynamic Structural Transformation for the Analysis of Distillation Control Structures
}

\author{
Moonyong Lee and Sunwon Park \\ Department of Chemical Engineering, KAIST, Seoul 305-701, Korea
}

\begin{abstract}
One of the most popular approaches to controlling multivariable systems in chemical processes may be to use multiloop SISO (single-input/single-output) control. One of the major concerns of this approach is to find the best control structure among various alternatives. Structural transformation is very useful for analyzing different control structures, because knowledge of the properties of any base control structure makes it possible to investigate various arbitrary structures without any further experiments or modeling. If the complete openloop transfer functions (COTF's) $G$ and $D$ of a base structure are available, transformation into an arbitrary structure can be made straightforwardly using the relationships between inputs of the base structure and inputs of the arbitrary structure. For distillation columns, however, it is difficult to find the COTF's experimentally, because slow composition dynamics and fast inventory dynamics exist simultaneously. When we make all the loops open and introduce a step change in a manipulated variable to find the COTF's, inventory outputs violate operational constraints long before composition outputs approach the steady state.
\end{abstract}

For this reason, the modified open-loop transfer functions (MOTF's) have been used commonly instead of the COTF's. By "modified open loop" we mean that composition loops are open while all inventory loops are closed. Indeed, most published dynamic models for distillation columns use the MOTF's. Another important reason to use the MOTF's is that there are only few pairing tools and decoupler design methods to handle the COTF's including integrating dynamics.

There have been several researches on transformation of distillation control structure (Skogestad and Morari, 1987; Häggblom and Waller, 1988, 1990; Yang et al., 1990). Häggblom and Waller $(1988,1990)$ presented a structural transformation method for perfect inventory control; however, Häggblom and Waller (1990) and Yang et al. (1990) have pointed out that when inventory control dynamics are included, this method is not valid except when inputs for inventory control are not changed by transformation. In addition, Häggblom and Waller do not explain clearly why their method is valid for the special cases. Yang et al. (1990) presented the extended method including inventory control dynamics based

Correspondence concerning this work should be addressed to S. Park. on the framework of Häggblom and Waller's method. Although Yang et al.'s method provides a good insight into the effects of internal flow dynamics, the relationships between inputs for inventory control and inputs for composition control should be modified whenever inputs for inventory control are changed by structural transformation.

In this work, a new framework based on the relationships between the COTF's and the MOTF's is presented for the structural transformation of distillation control structures. The framework provides another view of structural transformation for distillation control structures, which has certain theoretical advantages. Using the framework we suggest a simple method of the structural transformation for the general dynamic case including inventory control dynamics. We also demonstrate why Häggblom and Waller's method is valid for the special cases.

\section{Framework for Structural Transformation}

Consider a simple distillation column in Figure 1, which may be viewed as a $4 \times 4$ system by neglecting the pressure control. Four flows are used usually as inputs or manipulated variables (that is, the reflux flow $L$, the boilup $V$, the distillate flow $D$,

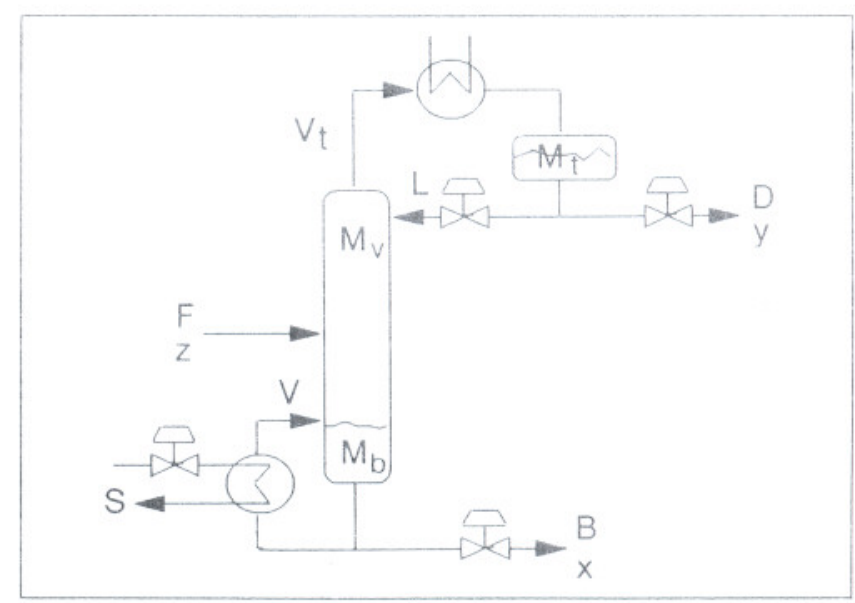

Figure 1. Two product distillation column. 
and the bottom flow $B$ ) to keep two inventory outputs (the receiver holdup $M_{t}$ and the base holdup $M_{b}$ ) and two composition outputs (the top composition $y$ and the bottom composition $x$ ). Two out of four inputs are used for inventory control and the other two for composition control. The feed flow $F$ and the feed composition $z$ acts as disturbances.

For a base structure, the complete open-loop relation among the output vector $Y$, the input vector $u$, and the disturbance vector $d$ is given by:

$$
Y=G u+D d
$$

Considering inventory and composition loops, we can rewrite Eq. 1 in the following block partitioned form:

$$
\left(\begin{array}{c}
Y_{c} \\
Y_{i}
\end{array}\right)=\left(\begin{array}{ccc}
G_{c c} & \vdots & G_{c i} \\
\hdashline G_{i c} & \vdots & G_{i i}
\end{array}\right)\left(\begin{array}{c}
u_{c} \\
\hdashline \\
u_{i}
\end{array}\right)+\left(\begin{array}{c}
D_{c} \\
\hdashline D_{i}
\end{array}\right) d
$$

where subscripts $c$ and $i$ stand for composition and inventory, respectively. Similarly, the complete open-loop relation for an arbitrary new structure is given by:

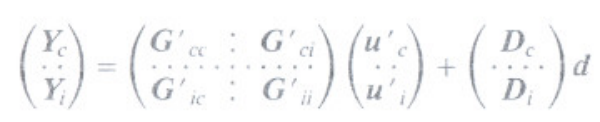

Note that $\boldsymbol{d}$ is independent of control structures while $u$ depends on control structures. The block element matrices $G_{i c}, G_{i i}$ and $D_{i}$ of the COTF's $G$ and $D$ include fast integrating dynamics, and can be obtained directly from plant tests. On the other hand, $\boldsymbol{G}_{c c}, \boldsymbol{G}_{c i}$, and $\boldsymbol{D}_{c}$ have much slower nonintegrating dynamics. Therefore, it is difficult to find $G_{c c}, G_{c i}$, and $D_{c}$ experimentally, because $Y_{i}$ violates inventory constraints before $Y_{c}$ approaches the steady state when step changes in $u$ or $d$ are introduced.

For this reason, the modified open-loop system shown in Figure 2 is considered. For the base structure, modified openloop relationships among the composition output vector $Y_{c}$, the composition input vector $u_{c}$, and $d$ are represented using the MOTF's $\boldsymbol{L}_{c c}$ and $\boldsymbol{L}_{c d}$ as follows:

$$
\boldsymbol{Y}_{c}=\boldsymbol{L}_{c c} \boldsymbol{u}_{c}+\boldsymbol{L}_{c d} \boldsymbol{d}
$$

where

$$
\boldsymbol{L}_{c c}=\left(\partial Y_{c} / \partial u_{c}\right)_{d} \text { and } \boldsymbol{L}_{c d}=\left(\partial Y_{c} / \partial d\right)_{u_{c}} .
$$

Similarly, modified open-loop relationships for the new structure are represented using the new MOTF's $L_{c c}^{\prime}$ and $\mathbf{L}_{c d}^{\prime}$ as follows:

$$
Y_{c}=L^{\prime}{ }_{c c} u^{\prime}{ }_{c}+L^{\prime}{ }_{c d} d
$$

where

$$
\boldsymbol{L}_{c c}^{\prime}=\left(\partial Y_{c} / \partial u_{c}^{\prime}\right)_{d} \text { and } L_{c d}^{\prime}=\left(\partial Y_{c} / \partial d\right)_{u}{ }_{c}{ }_{c}
$$

By structural transformation what we are trying to accomplish is to obtain $\boldsymbol{L}^{\prime}{ }_{c c}$ and $\boldsymbol{L}^{\prime}{ }_{c d}$ from $\boldsymbol{L}_{c c}, \boldsymbol{L}_{c d}$, and some additional

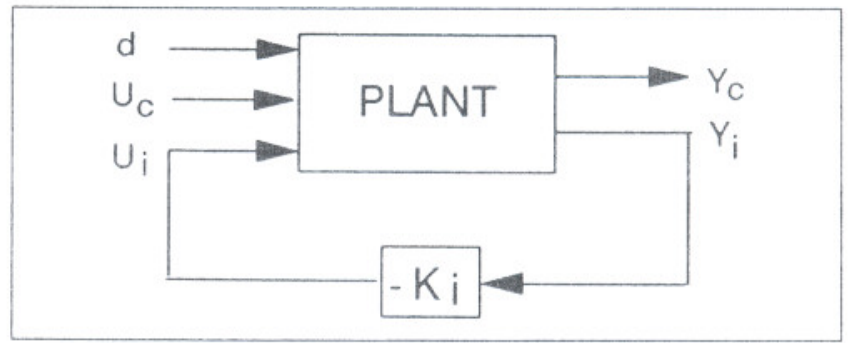

Figure 2. Conceptual diagram for the modified openloop system.

information of the base structure. For example, Häggblom and Waller $(1988,1990)$ used the relations among inputs for inventory control, inputs for composition control, and disturbances as the additional information:

$$
u_{i}=\boldsymbol{M}_{i c} \boldsymbol{u}_{c}+\boldsymbol{M}_{i d} \boldsymbol{d}
$$

where

$$
M_{i c}=\left(\partial u_{i} / \partial u_{c}\right)_{d} \text { and } M_{i d}=\left(\partial u_{i} / \partial d\right)_{u} .
$$

Figure 3 shows the block diagram for the modified openloop system in Figure 2. If $u^{\prime}$ and $u$ are related by the transformation matrix $\boldsymbol{H}$ as:

$$
\left(\begin{array}{c}
u^{\prime}{ }_{c} \\
\hdashline u_{i}^{\prime}
\end{array}\right)=\left(\begin{array}{lll}
H_{c c} & \vdots & H_{c i} \\
\hdashline H_{i c} & \vdots & H_{i i}
\end{array}\right)\left(\begin{array}{c}
u_{c} \\
\hdashline \\
u_{i}
\end{array}\right)
$$

then, from Eqs. 3 and 7, Figure 3 can also be represented in terms of $G^{\prime}, D, H$ and $K_{i}$ as shown in Figure 4 . These equivalent block diagrams provide a clear insight into the principle of - structural transformation. Based on Figure 4 we investigated the relations between the COTF's and the MOTF's. $Y_{c}$ can be regarded as the function of $u_{c}, d$ and $u_{c}{ }_{c}$, that is, $Y_{c}=Y_{c}\left(u_{c}\right.$, $d, u^{\prime}{ }_{c}$ ) where $u_{c}$ and $d$ are independent variables while $u^{\prime}{ }_{c}$ depends on both $u_{c}$ and $\boldsymbol{d}$. Note that regarding $Y_{c}$ as the function of $\boldsymbol{u}^{\prime}{ }_{c}$ makes it easy to analyze the relationships between the MOTF's used in Häggblom and Waller's approach and the COTF's. Applying the chain rule of partial differentiation gives:

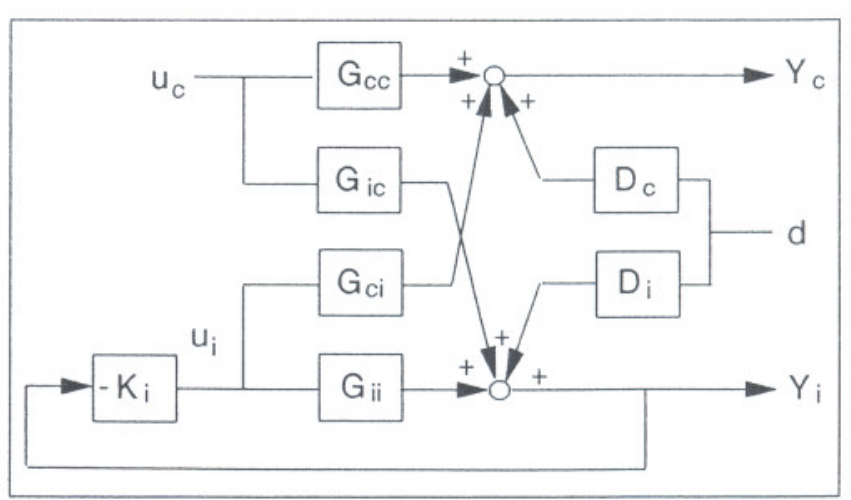

Figure 3. Modified open-loop system represented in terms of $G$ and $D$. 


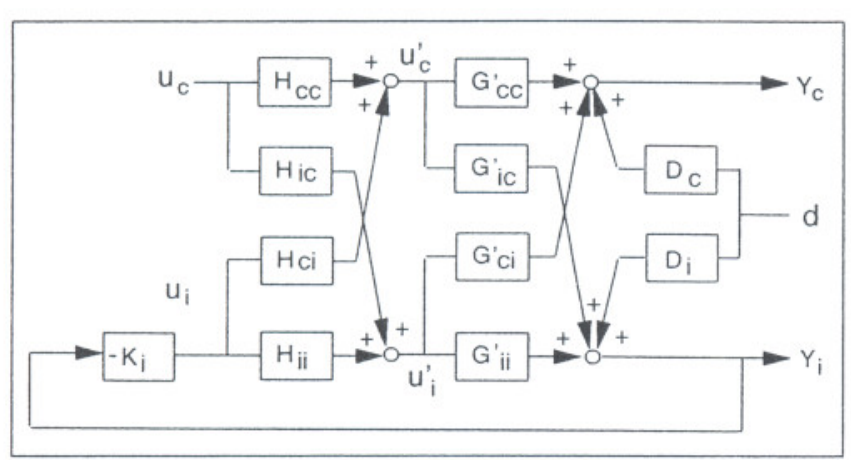

Figure 4. Modified open-loop system represented in terms of $H, G^{\prime}$, and $D$.

$$
\begin{gathered}
\left(\partial Y_{c} / \partial u_{c}\right)_{d}=\left(\partial Y_{c} / \partial u_{c}\right)_{d, u^{\prime}{ }_{c}}+\left(\partial Y_{c} / \partial u^{\prime}{ }_{c}\right)_{d, u_{c}}\left(\partial u^{\prime}{ }_{c} / \partial u_{c}\right)_{d} \\
\left(\partial Y_{c} / \partial d\right)_{u_{c}}=\left(\partial Y_{c} / \partial d\right)_{u_{c}, u_{c}^{\prime}{ }_{c}}+\left(\partial Y_{c} / \partial u^{\prime}{ }_{c}\right)_{d, u_{c}}\left(\partial u^{\prime}{ }_{c} / \partial d\right)_{u_{c}}
\end{gathered}
$$

The relations between the MOTF's of the base structure and the COTF's of the new structure can now be derived from Eqs. 8 and 9. From Figure 4 and the definitions of the MOTF's $\boldsymbol{L}_{c c}, \boldsymbol{L}_{c d}, \boldsymbol{M}_{i c}$, and $\boldsymbol{M}_{i d}$, Eqs. 8 and 9 become:

$$
\begin{aligned}
\boldsymbol{L}_{c c}= & \left\{G^{\prime}{ }_{c i}\left(\boldsymbol{I}+\boldsymbol{H}_{i i} \boldsymbol{K}_{i} G^{\prime}{ }_{i i}\right)^{-1} \boldsymbol{H}_{i c}\right\} \\
+ & \left\{G^{\prime}{ }_{c c}-G^{\prime}{ }_{c i}\left(\boldsymbol{I}+\boldsymbol{H}_{i i} \boldsymbol{K}_{i} G^{\prime}{ }_{i i}\right)^{-1} \boldsymbol{H}_{i i} \boldsymbol{K}_{i} G^{\prime}{ }_{i c}\right\}\left(\boldsymbol{H}_{c c}+\boldsymbol{H}_{c i} \boldsymbol{M}_{i c}\right) \\
\boldsymbol{L}_{c d}= & \left\{\boldsymbol{D}_{c}-G^{\prime}{ }_{c i}\left(\boldsymbol{I}+\boldsymbol{H}_{i i} \boldsymbol{K}_{i} G^{\prime}{ }_{i i}\right)^{-1} \boldsymbol{H}_{i i} \boldsymbol{K}_{i} \boldsymbol{D}_{i}\right\} \\
& +\left\{G^{\prime}{ }_{c c}-G^{\prime}{ }_{c i}\left(\boldsymbol{I}+\boldsymbol{H}_{i i} \boldsymbol{K}_{i} G^{\prime}{ }_{i i}\right)^{-1} \boldsymbol{H}_{i i} \boldsymbol{K}_{i} \boldsymbol{G}^{\prime}{ }_{i c}\right\}\left(\boldsymbol{H}_{c i} \boldsymbol{M}_{i d}\right)
\end{aligned}
$$

The relations between the MOTF's of the new structure and the COTF's of the new structure can also be derived from Eqs.

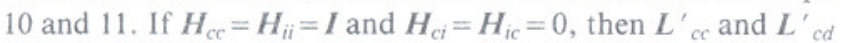
are identical to $\boldsymbol{L}_{c c}$ and $\boldsymbol{L}_{c d}$, respectively. Substituting these conditions into Eqs. 10 and 11 yields:

$$
\begin{gathered}
\boldsymbol{L}_{c c}^{\prime}=\boldsymbol{G}^{\prime}{ }_{c c}-\boldsymbol{G}^{\prime}{ }_{c i}\left(\boldsymbol{I}+\boldsymbol{K}_{i} \boldsymbol{G}^{\prime}{ }_{i i}\right)^{-1} \boldsymbol{K}_{i} \boldsymbol{G}^{\prime}{ }_{i c} \\
\boldsymbol{L}_{c d}^{\prime}=\boldsymbol{D}_{c}-\boldsymbol{G}^{\prime}{ }_{c i}\left(\boldsymbol{I}+\boldsymbol{K}_{i} \boldsymbol{G}^{\prime}{ }_{i i}\right)^{-1} \boldsymbol{K}_{i} \boldsymbol{D}_{i}
\end{gathered}
$$

\section{Transformation for the General Case}

In the general case where inventory control dynamics are included, as seen from Eqs. 10-13, it is difficult to obtain the MOTF's of the new structure $\left(\boldsymbol{L}^{\prime}{ }_{c c}\right.$ and $\left.\boldsymbol{L}^{\prime}{ }_{c d}\right)$ from the MOTF's of the base structure $\left(\boldsymbol{L}_{c c}, \boldsymbol{L}_{c d}, \boldsymbol{M}_{i c}\right.$, and $\left.\boldsymbol{M}_{i d}\right)$ alone. Thus, Häggblom and Waller's method is no longer valid. Fortunately, however, the peculiar characteristics of distillation columns make the situations somewhat simple if the $L V$ structure is chosen as the base structure.

If we choose the $L V$ structure as the base structure, then $G_{c i}$ becomes a null matrix because $u_{i}(D$ and $B$ ) has no effect on $Y_{c}(y$ and $x)$ when the mixing dynamics in the accumulators are neglected. Therefore, from Figure 3, we can see that $L_{c c}$ and $\boldsymbol{L}_{c d}$ are identical with $\boldsymbol{G}_{c c}$ and $\boldsymbol{D}_{c}$, respectively.

$$
\begin{aligned}
& \boldsymbol{G}_{c c}=\boldsymbol{L}_{c c} \\
& \boldsymbol{D}_{c}=\boldsymbol{L}_{c d}
\end{aligned}
$$

Therefore, we can determine $\boldsymbol{G}_{c c}$ and $\boldsymbol{D}_{c}$ by obtaining $\boldsymbol{L}_{c c}$ and $\boldsymbol{L}_{c d}$ experimentally. Furthermore, we can also obtain $\boldsymbol{G}_{i c}, \boldsymbol{G}_{i i}$, and $\boldsymbol{D}_{i}$ experimentally. Thus, the COTF's $\boldsymbol{G}$ and $\boldsymbol{D}$ can be completely determined by knowing $\boldsymbol{L}_{c c}, \boldsymbol{L}_{c d}, \boldsymbol{G}_{i c}, \boldsymbol{G}_{i i}$, and $\boldsymbol{D}_{i}$. Once $\boldsymbol{G}$ and $\boldsymbol{D}$ are determined, structural transformation into any new structure is straightforward. If $\boldsymbol{u}$ and $\boldsymbol{u}^{\prime}$ are related by the transformation matrix $\boldsymbol{P}$ as:

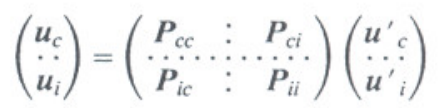

then the MOTF's $L^{\prime}{ }_{c c}$ and $L^{\prime}{ }_{c d}$ of the new structure can be derived from block diagram algebra as follows:

$$
\begin{gathered}
\boldsymbol{L}_{c c}^{\prime}=\boldsymbol{G}_{c c} \boldsymbol{P}_{c c}-\boldsymbol{G}_{c c} \boldsymbol{P}_{c i} \boldsymbol{S}^{\prime}{ }_{i}\left(\boldsymbol{G}_{i c} \boldsymbol{P}_{c c}+\boldsymbol{G}_{i i} \boldsymbol{P}_{i c}\right) \\
\boldsymbol{L}^{\prime}{ }_{c d}=\boldsymbol{D}_{c}-\boldsymbol{G}_{c c} \boldsymbol{P}_{c i} \boldsymbol{S}^{\prime}{ }_{i} \boldsymbol{D}_{i}
\end{gathered}
$$

where

$$
\boldsymbol{S}_{i}^{\prime}=\boldsymbol{K}_{i}\left\{\boldsymbol{I}+\left(\boldsymbol{G}_{i c} \boldsymbol{P}_{c i}+\boldsymbol{G}_{i i} \boldsymbol{P}_{i i}\right) \boldsymbol{K}_{i}\right\}^{-1}
$$

Note that for the case where the nonlinear relationships among inputs are desired (for example, the ratio of flow rates) $\boldsymbol{P}$ becomes partial derivatives at the nominal operation condition.

\section{Transformation for the Special Cases}

In this section, using the relationships between the COTF's and the MOTF's we analyze Häggblom and Waller's method and demonstrate the validity of their method for the special cases.

\section{Inventory loops perfectly controlled}

Perfect inventory control may be often a good approximation since the inventory dynamics are much faster than the composition dynamics. For the perfect inventory control case, Eqs. 12 and 13 reduce to:

$$
\begin{gathered}
\boldsymbol{L}_{c c}^{\prime}=\boldsymbol{G}_{c c}^{\prime}-G^{\prime}{ }_{c i} G^{\prime}{ }_{i i}^{-1} G^{\prime}{ }_{i c} \\
L_{c d}^{\prime}=D_{c}-G^{\prime}{ }_{c i} G^{\prime}{ }_{i i}^{-1} D_{i}
\end{gathered}
$$

Furthermore, the terms in Eqs. 8 and 9 become:

$$
\begin{gathered}
\left(\partial \boldsymbol{Y}_{c} / \partial u_{c}\right)_{d, u^{\prime}}=0 \\
\left(\partial Y_{c} / \partial u_{c}^{\prime}\right)_{d, u_{c}}=G_{c c}^{\prime}-G_{c i}^{\prime} G_{i i}^{\prime-1} G_{i c}^{\prime} \\
\left(\partial Y_{c} / \partial d\right)_{u_{c, u^{\prime}{ }_{c}}}=D_{c}-G_{c i}^{\prime} G_{i i}^{\prime}{ }^{-1} D_{i}
\end{gathered}
$$

Using the results of Eqs. 20-24, we can rewrite Eqs. 10 and 11 more compactly as:

$$
\begin{aligned}
& \boldsymbol{L}_{c c}=\boldsymbol{L}_{c c}^{\prime}\left(\boldsymbol{H}_{c c}+\boldsymbol{H}_{c i} \boldsymbol{M}_{i c}\right) \\
& \boldsymbol{L}_{c d}=\boldsymbol{L}_{c d}^{\prime}+\boldsymbol{L}_{c c}^{\prime} \boldsymbol{H}_{c i} \boldsymbol{M}_{i d}
\end{aligned}
$$

Equations 25 and 26 are readily rearranged as follows: 
Table 1. UCSB Simulation Model of $L V$ Structure for Impurity Control*

$$
\begin{gathered}
Y_{c}=\left[\begin{array}{c}
\Delta y \\
\Delta x
\end{array}\right], \quad Y_{i}=\left[\begin{array}{c}
\Delta M_{t} \\
\Delta M_{b}
\end{array}\right], \quad u_{c}=\left[\begin{array}{l}
\Delta L \\
\Delta V
\end{array}\right], \quad u_{i}=\left[\begin{array}{c}
\Delta D \\
\Delta B
\end{array}\right], \quad d=\left[\begin{array}{c}
\Delta F \\
\Delta z
\end{array}\right] \\
L_{c c}=\left[\begin{array}{cc}
\frac{-3.58 e^{-2 \cdot 1 s}}{19.2 s+1} & \frac{3.38 e^{-2 \cdot 2 s}}{19.8 s+1} \\
\frac{13.9 e^{-1 \cdot 2 s}}{13.2 s+1} & \frac{-18.9 e^{-0.6 s}}{12.6 s+1}
\end{array}\right] \quad L_{c d}=\left[\begin{array}{cc}
\frac{-0.515 e^{-3.75}}{27.6 s+1} & \frac{23.1 e^{-4 \cdot 9 s}}{22.8 s+1} \\
\frac{9.97 e^{-0.4 s}}{10.8 s+1} & \frac{-213 e^{-1.5 s}}{14.5 s+1}
\end{array}\right] \\
G_{i c}=\left[\begin{array}{cc}
\frac{-0.775}{s} & \frac{0.818}{s} \\
\frac{0.775}{s} & \frac{-0.818}{s}
\end{array}\right] \quad G_{i i}=\left[\begin{array}{cc}
\frac{-1.0}{s} & 0 \\
0 & \frac{-1.0}{s}
\end{array}\right] \quad D_{i}=\left[\begin{array}{cc}
\frac{0.038}{s} & \frac{-1.2}{s} \\
\frac{0.962}{s} & \frac{1.2}{s}
\end{array}\right] \quad K_{i}=\left[\begin{array}{cc}
\frac{-1.0}{1.14} & 0 \\
0 & \frac{-1.0}{1.75}
\end{array}\right]
\end{gathered}
$$

- Time constants and time delays are expressed in minutes; all inventory-controlled variables are in terms of moles; the unit of $P$-controller is min ${ }^{-1}$; all composition gains have been multiplied by $10^{3}$

$$
\begin{aligned}
& \boldsymbol{L}_{c c}^{\prime}=\boldsymbol{L}_{c c}\left(\boldsymbol{H}_{c c}+\boldsymbol{H}_{c i} \boldsymbol{M}_{i c}\right) \\
& L_{c d}^{\prime}=L_{c d}-L^{\prime}{ }_{c c} H_{c i} M_{i d}
\end{aligned}
$$

The results show that $L^{\prime}{ }_{c c}$ and $L^{\prime}{ }_{c d}$ can be obtained from $L_{c c}$, $\boldsymbol{L}_{c d}, \boldsymbol{M}_{i c}$, and $\boldsymbol{M}_{i d}$. This approach clearly explains why Häggblom and Waller's method is valid under the assumptions of steady state or perfect inventory control.

\section{Inputs for inventory control unchanged}

Consider the case where inventory control dynamics are included, but inputs for inventory control are not changed by transformation $\left(\boldsymbol{H}_{i c}=0\right.$ and $\left.\boldsymbol{H}_{i i}=\boldsymbol{I}\right)$. Substituting $\boldsymbol{H}_{i c}=0$ and $H_{i i}=I$ into Eqs. 10 and 11 , and using Eqs. 12 and 13, we can easily show that Eqs. 10 and 11 readily reduce to Eqs. 25 and 26 , respectively. Thus, Häggblom and Waller's approach is still valid.

\section{Example: Transformation from LV to DV Structure}

The dynamic model based on the UCSB simulation model (Yang, 1991) was used to confirm the proposed method. The model is shown in Table 1. $\boldsymbol{G}_{i c}, \boldsymbol{G}_{i i}, \boldsymbol{D}_{i}$ and $\boldsymbol{K}_{i}$ were derived from $\boldsymbol{M}_{i c}, \boldsymbol{M}_{i d}$, and the parameters of $P$-controllers in the Yang model (1991) by using the properties of internal flow dynamics in distillation columns. For simplicity, holdups in terms of moles are used for inventory control loops instead of levels used in the original work by Yang et al. (1990).

Consider transformation from $L V$ structure to $D V$ structure. $u$ and $u^{\prime}$ are:

$$
u_{c}=\left(\begin{array}{l}
\Delta L \\
\Delta V
\end{array}\right), u_{i}=\left(\begin{array}{l}
\Delta D \\
\Delta B
\end{array}\right), u_{c}^{\prime}=\left(\begin{array}{l}
\Delta D \\
\Delta V
\end{array}\right), u_{i}^{\prime}=\left(\begin{array}{l}
\Delta L \\
\Delta B
\end{array}\right)
$$

Thus, the transformation matrix $P$ is:

$$
P_{c c}=P_{i i}=\left(\begin{array}{ll}
0 & 0 \\
0 & 1
\end{array}\right), P_{c i}=P_{i c}=\left(\begin{array}{ll}
1 & 0 \\
0 & 0
\end{array}\right)
$$

- $L^{\prime}{ }_{c c}$ and $L^{\prime}{ }_{c d}$ obtained by the proposed method are given in Table 2. The open-loop responses of the $D V$ structure were also investigated using the MOTF's obtained by structural transformation for the two cases: 1 . the case with the assumption of perfect inventory control; 2 ., the case without the assumption of perfect inventory control. Figure 5 shows the open-loop responses of the top and bottom compositions to a step change of $\Delta V=0.1 \mathrm{~mol} / \mathrm{min}$. The dynamic responses with and without the assumption of perfect inventory control are different signficantly. In this case, we may have severe troubles when we design composition control loops based on the perfect inventory control.

Table 2. MOTF's of $D V$ Structure Obtained by Structural Transformation

$$
\begin{aligned}
{[\Delta y} & \Delta x]= \\
& {\left[\begin{array}{ll}
\frac{4.62 e^{-2 \cdot 1 s}}{(19.2 s+1)(1.47 s+1)} & \frac{3.38 e^{-2 \cdot 2 s}}{(19.8 s+1)}+\frac{-3.78 e^{-2 \cdot 1 s}}{(19.2 s+1)(1.47 s+1)} \\
\frac{-17.9 e^{-1 \cdot 2 s}}{(13.2 s+1)(1.47 s+1)} & \frac{-18.9 e^{-0.6 s}}{(12.6 s+1)}+\frac{14.7 e^{-1 \cdot 2 s}}{(13.2 s+1)(1.47 s+1)}
\end{array}\right]\left[\begin{array}{c}
\Delta D \\
\Delta V
\end{array}\right] } \\
& +\left[\begin{array}{cc}
\frac{-0.515 e^{-3 \cdot 7 s}}{(27.6 s+1)}+\frac{-0.176 e^{-2 \cdot 1 s}}{(19.2 s+1)(1.47 s+1)} & \frac{23.1 e^{-4 \cdot 9 s}}{(22.8 s+1)}+\frac{5.54 e^{-2 \cdot 1 s}}{(19.2 s+1)(1.47 s+1)} \\
\frac{9.97 e^{-0.4 s}}{(10.8 s+1)}+\frac{0.685 e^{-1 \cdot 2 s}}{(13.2 s+1)(1.47 s+1)} & \frac{-213 e^{-1 \cdot 5 s}}{(14.5 s+1)}+\frac{-21.5 e^{-1 \cdot 2 s}}{(13.2 s+1)(1.47 s+1)}
\end{array}\right]\left[\begin{array}{c}
\Delta F \\
\Delta z
\end{array}\right]
\end{aligned}
$$



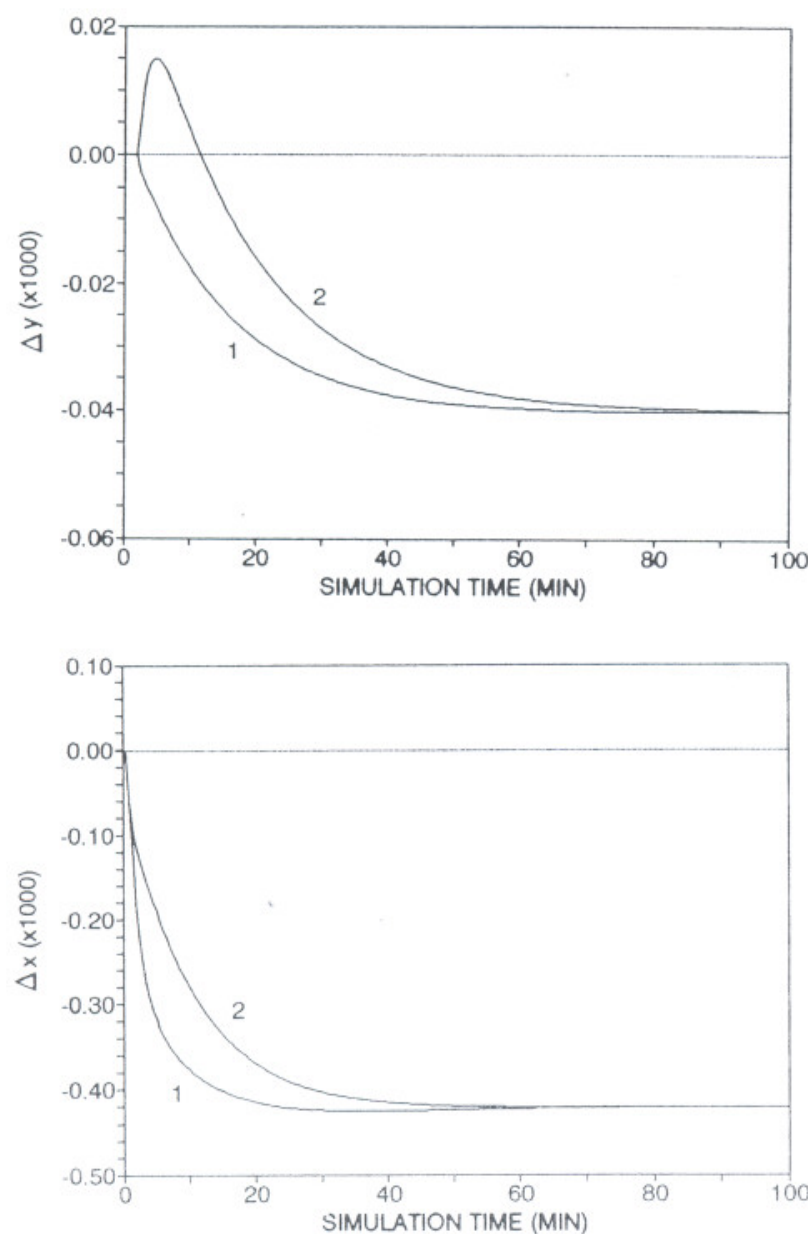

Figure 5. Open-loop responses of $D V$ structure for a step change of $\Delta V=0.1 \mathrm{~mol} / \mathrm{min}$ using the MOTF's obtained by structural transforma. tion.

1. with the assumption of perfect inventory control; 2 . without the assumption of perfect inventory control.

\section{Acknowledgment}

The authors gratefully acknowledge financial support from the Yukong Limited and the Korea Ministry of Science and Technology. We also would like to thank Professor Duncan A. Mellichamp who reviewed this work and Dr. Dae R. Yang for their valuable comments.

\section{Notation}

$B=$ bottom flow rate, $\mathrm{mol} / \mathrm{min}$

$\boldsymbol{d}=$ disturbance vector

$\boldsymbol{D}=$ complete open-loop transfer function between disturbances and outputs where all inventory and composition loops are open

$D=$ distillate flow rate, $\mathrm{mol} / \mathrm{min}$

$F=$ feed flow rate, $\mathrm{mol} / \mathrm{min}$

$\boldsymbol{G}=$ complete open-loop transfer function between inputs and outputs where all inventory and composition loops are open

$H=$ transformation matrix

$I=$ identity matrix

$\boldsymbol{K}_{i}=$ controller for inventory loops, $\mathrm{min}^{-1}$

$L=$ reflux flow rate, $\mathrm{mol} / \mathrm{min}$

$\boldsymbol{L}=$ modified open-loop transfer function between inputs (or disturbances) and outputs where inventory loops are closed

$\boldsymbol{M}=$ modified open-loop transfer function between inputs for composition control (or disturbances) and inputs for inventory control where inventory loops are closed

$\boldsymbol{M}_{t}, \boldsymbol{M}_{b}=$ liquid holdup in the receiver and the base, respectively, mol

$P=$ transformation matrix

$\boldsymbol{u}=$ input or manipulated variable vector

$V=$ boilup rate, $\mathrm{mol} / \mathrm{min}$

$x=$ bottom composition, mol fraction

$y=$ top composition, mol fraction

$Y=$ output vector or controlled variable vector

$z=$ feed composition, mol fraction

\section{Superscripts}

$=$ new control structure

\section{Subscripts}

$$
c=\text { index for composition }
$$

$i=$ index for inventory

\section{Literature Cited}

Häggblom, K. E., and K. V. Waller, "Transformation and Consistency Relations of Distillation Control Structures," AIChE J., 34, 1634 (1988).

Häggblom, K. E., and K. V. Waller, "Control Structures for Disturbance Rejection and Decoupling of Distillation," AIChE J., 36, 1107 (1990).

Skogestad, S., and M. Morari, "Control Configuration Selection for Distillation Columns," AlChE J., 33, 1620 (1987).

Yang, D. R., personal communication (1991).

Yang, D. R., K. V. Waller, D. E. Seborg, and D. A. Mellichamp, "Dynamic Structural Transformations for Distillation Control Configurations," AIChE J., 36, 1391 (1990).

Manuscript received Mar. 25, 1991, and revision received Aug. 29, 1991. 